\title{
Correction: Crop breeding: Turning a lawn into a field
}

Richard Sibout

Nature Plants 3, 17060 (2017); published 18 April 2017; corrected 20 April 2017.

When this News \& Views was originally published, the website stated the wrong publication date. This News \& Views was in fact published on 18 April 2017. 